\section{Practical considerations when treating pets of owners with no fixed abode: treatment priorities, implementation and compliance}

\section{Fraje Watson}

Pets are vitally important to their homeless owners and, as such, they are extremely well cared for despite the difficulties they face such as stigma and expense. Owners experiencing homelessness often need help providing food, collars, leads, toys and preventative healthcare for their pets. Veterinary charities that are established to work with homeless and vulnerable people can help provide these basic provisions, as well as providing trusted and dependable veterinary care.

Veterinary care to these pets is usually done on the roadside in public areas, which can cause barriers to treatment that need to be overcome. Teams of three to five veterinary professionals consult in groups consisting of veterinary surgeons and registered veterinary nurses. The team carry an army surplus backpack containing core diagnostic equipment, paperwork and medication. Owners of new patients often need help ensuring they comply with legal requirements such as; provision of a lead and collar including identification tag, microchipping and access to poo bags. For some patients it might be necessary to take steps to reduce the risk of antisocial behaviour and its consequences. Additional priorities to consider include; health problems that might affect their volatile housing situation (e.g. ectoparasites, diarrhoea), other behavioural issues (e.g. dog aggression, separation anxiety) and zoonoses.

A full clinical examination can be challenging in a busy, public environment particularly with nervous dogs or cats - or any species that requires an eye examination! Once a diagnosis has been made, consideration of the treatment plan and follow-up examinations must be considered. Only certain medications are carried in the backpack, though most medications can be accessed via veterinary practice partners with a few days' notice, whilst other medications either cannot be dispensed at all (e.g. controlled drugs) or cannot be stored correctly by the owner (e.g. refrigerated items). Where cage rest is required additional dispensation may need to be made such as prolonged hospital stays or foster stays, as pets are unlikely to receive this in their usual environment. Many owners will attend sessions weekly, whether their pet requires veterinary treatment or not, whilst others may not be relied upon to attend follow-up appointments. Despite these challenges, owners can be expected to be devoted and provide hands-on, roundthe-clock care for their pet (e.g. 2-hourly eye drops).

Owner compliance requires consideration as they may live hectic or unreliable lifestyles with social challenges of their own, or a pet may be looked after by multiple people. Owner health status must be carefully considered as poor personal health is common in owners of no fixed abode, and they may not be forthcoming with their particular difficulties. When prescribing medication or treatment plans it is important to consider that an owner may be blind or have poor eyesight without access to glasses, they may have limited or no ability to read and they may struggle with their memory.

Providing veterinary care to the pets of homeless and vulnerable people has a specific set of challenges that often require additional planning and forethought amongst the veterinary team.

\section{KEY LEARNING OBJECTIVES}

- Understanding that it is essential that pets of owners of no fixed abode meet the legal requirements of pet ownership (e.g. microchipping)

- Appreciate common medications required in this population, how medications can be stored, equipment required for a full clinical examination and disposables necessary for diagnostic tests

- Understand that the unique environment of each owner must be considered in order to adapt medications and treatments as necessary

\section{MULTIPLE CHOICE QUESTIONS}

1. What information is legally required to be on a dog's collar whilst on a highway or public place?
(A) Dog's name, owner's name, address, telephone number
(B) Owner's name, owner's address, telephone number
(C) Owner's name, owner's address
(D) Dog's name, owner's name

2. What temperature should medication in the backpack be stored at?
(A) $8-25^{\circ} \mathrm{C}$
(B) $5-20^{\circ} \mathrm{C}$
(C) $1-5^{\circ} \mathrm{C}$
(D) $12-30^{\circ} \mathrm{C}$

3. What information does NOT need to be included on the label of dispensed POM-V medication according to the RCVS recommendations?
(A) 'For animal treatment only'
(B) Name of the dispensing veterinary surgeon
(C) Date of supply
(D) Name, strength and quantity of medication 